\title{
Pais/Cuidadores Com e Sem Histórico de Abuso: Punições Corporais e Características Psicológicas
}

\author{
Roberta Noronha Azevedo ${ }^{1}$ \\ ${ }^{1}$ Universidade de São Paulo, SP, Brasil.
}

\author{
Marina Rezende Bazon \\ ${ }^{1}$ Universidade de São Paulo, SP, Brasil.
}

Resumo: A prevalência dos castigos físicos em crianças/adolescentes é muito elevada. Sua aceitação social é ampla, sendo que as legislações brasileiras criam brechas para sua ocorrência. Entre os cientistas, há dissenso quanto aos limites entre castigos e abusos físicos. O objetivo do presente estudo foi contribuir com as discussões nesse âmbito, buscando verificar se grupos de pais/responsáveis sem e com histórico de notificação ao sistema de proteção por abuso físico se diferenciariam quanto à forma de punir/castigar e quanto a variáveis psicossociais consideradas fatores de risco para abuso físico. A abordagem adotada no estudo foi quantitativa, com delineamento transversal e comparativo. Participaram do estudo pais/ cuidadores que faziam utilização da punição corporal: 47 sem histórico de notificação e 40 com histórico de notificação por abuso físico. Os instrumentos utilizados para a coleta de dados foram: Questionário de Caracterização Sociodemográfica, Roteiro de Entrevista sobre Prática Parental, Inventário de Potencial de Maus-Tratos Infantil (Child Abuse Potential Inventory-CAP). Os pais notificados relataram punições corporais mais graves (ou seja, mais frequentes, mais gravosas e em regiões do corpo mais vulneráveis) e mais angústia, rigidez, infelicidade, problemas com a criança, problemas com os outros e solidão. Todavia, em ambos os grupos, segundo o reportado, a raiva moveria o ato de punir. Esses resultados reiteram a ideia de que todas as formas de punição física são maus-tratos (expressões de violência), pois decorrem das necessidades dos cuidadores ("descarga emocional"). Remetem, porém, a um fenômeno heterogêneo, em termos de gravidade e de fatores associados, que precisa ser assim considerado na proposição de programas de prevenção e de tratamento.

Palavras-chave: Punição, Abuso da Criança, Estilo Parental, Violência na Família.

\section{Parents/Caregivers With and Without History of Abuse: Corporal Punishment and Psychological Characteristics}

\begin{abstract}
Physical punishment of children / adolescents is highly prevalent and socially accepted, with a series of loopholes in the Brazilian legislation that favor this practice. Scientists tend to disagree in regard to the limits between physical punishment and physical abuse. This study aimed to contribute to the discussions around this theme, verifying whether practices of physical punishment and psychosocial variables considered risk factors for physical abuse differ among parents / caregivers with and without history of physical abuse notifications to protection agencies. The study was conducted with parents / caregivers who adopt practices of corporal punishment, 47 of whom without and 40 with history of physical abuse notification. Data were collected by means of Characterization Questionnaire, Parental Practice Interview, and Child Abuse Potential Inventory (CAP). Notified parents reported more severe corporal punishments (more frequent, more dangerous, in more vulnerable body regions) and more distress, severity, unhappiness, problems with the child and other people, and loneliness. Both groups reported that the act of punishing was moved by anger. These results reiterate the notion that all forms
\end{abstract}


of physical punishment are maltreatment (expressions of violence) resulting from the needs of the caregivers ("emotional discharge"). However, these practices refer to a heterogeneous phenomenon in terms of severity and associated factors, which must be considered when proposing prevention and treatment programs.

Keywords: Punishment, Violence Against Children, Parental Style, Family Violence.

\title{
Padres/Cuidadores Con y Sin Antecedentes de Maltrato: Castigos Corporales y Características Psicológicas
}

\begin{abstract}
Resumen: Los castigos corporales de niños/adolescentes tiene una prevalencia muy elevada. Su aceptación social es amplia, y las legislaciones brasileñas no logran abarcar las puniciones adecuadas para esta práctica. No existe un consenso entre los científicos en cuanto a los límites entre punición corporal y maltrato físico. El objetivo del presente estudio fue contribuir con las discusiones sobre ese tema, verificando si grupos de padres/responsables sin y con antecedentes de notificación al Sistema de Protección por maltrato físico se diferenciarían en la forma de castigar/punir y en variables psicosociales consideradas factores de riesgo para maltrato físico. Este es un estudio cuantitativo, de tipo transversal y comparativo. Participaron en el estudio padres/cuidadores que hacían uso del castigo corporal: 47 sin antecedentes de notificación al sistema; 40 con antecedentes de notificación por maltrato físico. Los instrumentos utilizados para la recolección de datos fueron: Cuestionario de Datos Sociodemográficos, Entrevista sobre Práctica Parental, Inventario de Potencial de Maltrato Infantil (Child Abuse Potential Inventory - CAP). Los padres notificados reportaron castigos corporales más graves (es decir, más frecuentes, más graves, en regiones del cuerpo más vulnerables) y más angustia, rigidez,infelicidad, problemas con el niño, problemas conlos demásysoledad. Según el reportado, en ambos grupos, la rabia movería el acto de castigar. Estos resultados reiteran la idea de que todas las formas de castigo físico son maltrato (manifestación de violencia), pues se derivan de las necesidades de los cuidadores (“descarga emocional”). Sin embargo, remiten a un fenómeno heterogéneo, en términos de gravedad y de factores asociados, que necesita considerarse en la propuesta de programas de prevención y de tratamiento.
\end{abstract}

Palabras clave: Castigo, Maltrato de Niños, Creación de Hijos, Violencia Doméstica.

\section{Introdução}

Ao longo da história da humanidade, as crianças vêm sendo submetidas a diversas formas de violência (Organização Mundial da Saúde [OMS], 2016). Gradativamente, dada a maior sensibilidade à infância, essas situações passaram a preocupar a sociedade e, por volta da segunda metade do século XX, a academia científica também, de modo que o tema passou a ser investigado por pesquisadores de diferentes áreas (Durrant, Stewart-Tufescu, \& Afifi, 2019). Dentre os diversos tipos de violência, a de natureza física figura entre os mais estudados; boa parte da produção de conhecimento científico concernente à violência contra as crianças está voltada aos chamados abusos físicos, sobretudo aqueles praticados no âmbito doméstico (Gershoff \& Grogan-Kaylor, 2016).

Nessa seara, observa-se, contudo, que há um importante debate, tanto em termos sociais quanto acadêmicos, sobre o fato de a punição corporal/os castigos físicos e os abusos físicos serem equivalentes ou não, de forma que a falta de consenso, nesse tocante, configura um obstáculo ao estabelecimento de parâmetros legais claros, bem como ao avanço na produção de conhecimento científico na área (Durrant, Fallon, Lefebvre, \& Allan, 2017). Permanece aberto o espaço para a existência de diferentes opiniões sobre o assunto, tanto em meio a leigos como em meio a profissionais 
de diversas especialidades, que lidam direta ou indiretamente com o segmento infanto-juvenil.

No tocante à visão jurídica, no conjunto, as legislações criam algum nível de contradição com relação à admissibilidade da punição corporal em crianças (Durrant et al., 2019). Há, todavia, um crescente movimento internacional de promulgação de legislações específicas que proíbam o uso do castigo físico contra crianças (Ribeiro, 2016). Até 2018, eram 54 os países que possuíam leis com esse intuito, mas, em boa parte deles, permanecem ambiguidades legais que dão margem para a perpetuação social desse comportamento em relação às crianças (Durrant et al., 2017). No Brasil, a despeito dos novos referenciais para o tratamento de crianças e adolescentes estabelecidos na Constituição Federal (Constituição da República Federativa do Brasil [CF], 1988) e no Estatuto da Criança e do Adolescente - ECA (Lei n. 8.069/1990, 1990), pode-se afirmar que não há clareza com relação à temática. O Código Civil concebe que os pais, com o intuito de educar os filhos menores, podem utilizar em sua função correcional o direito de castigar seus filhos, o chamado jus corrigendi. Nesse âmbito, proíbe apenas o castigo físico aplicado de maneira "imoderada", ao dispor em seu artigo 1.638 sobre a perda do poder familiar (Lei n. 10.406/2002, 2002). Os juristas que se opõem a esse pensamento afirmam que a aceitação implícita do "castigo moderado" vai de encontro aos direitos fundamentais da pessoa humana. Nessa corrente, defende-se que todo castigo físico configura uma forma de violência, que corrompe a dignidade e o respeito a que todos têm direito (Durrant et al., 2019).

A chamada Lei Menino Bernardo (Lei n. 13.010/2014, 2014), vulgarmente conhecida como Lei da Palmada, teria sido editada para aclarar essas questões. Ela alterou o artigo 13 do ECA e introduziu novas redações aos artigos 18 e 70, em uma tentativa de melhor definir o que seriam "castigo físico" e "tratamento cruel e degradante", além de alterar as sanções previstas ao descumprimento da Lei. No $1^{\circ}$ artigo da Lei, estipula-se que as crianças e os adolescentes têm o direito de serem educados e cuidados sem o uso de castigo físico ou tratamento cruel ou degradante, como formas de correção, disciplina, educação ou qualquer outro pretexto, explanando o que seriam considerados "castigo físico" e "tratamento cruel ou degradante" da seguinte forma (Lei n. 13.010/2014, 2014):
I - castigo físico: ação de natureza disciplinar ou punitiva aplicada com o uso da força física sobre a criança ou o adolescente que resulte em:

a) sofrimento físico; ou

b) lesão;

II - tratamento cruel ou degradante: conduta ou forma cruel de tratamento em relação à criança ou ao adolescente que:

a) humilhe; ou

b) ameace gravemente; ou

c) ridicularize.

Especialistas em Direito divergem quanto à contribuição oferecida por esta lei. Alguns apontam que foi importante, principalmente, por aportar algum avanço com relação ao Código Civil, deixando claro que nem o castigo moderado nem o imoderado são permitidos (Dias, 2014). Outros sublinham que a Lei não proibiu de fato o castigo físico, mas apenas aqueles que resultem em "sofrimento físico" ou "lesões", e destacam que a grande questão, nessa esfera, é a ampla margem de manobra para aquilo que será considerado "sofrimento físico", uma vez que não se dispõe de qualquer critério objetivo para sua identificação/ mensuração, cabendo aos juízes, no uso do poder discricionário, definir tal conceito, baseados no contexto de cada situação (Rodrigues \& Tomé, 2014).

Semelhante ao que ocorre no meio jurídico, nota-se, no âmbito acadêmico, a existência de divergências. Há quem defenda que os conceitos remetem a conjuntos de ações diferentes, associadas a variáveis também diferenciadas, sendo as punições corporais um fator de risco aos abusos (Afifi et al., 2017; Gershoff \& Grogan-Kaylor, 2016). Argumenta-se que, embora ambas as ações possam ser consideradas violências, elas são diferenciáveis em termos de menor ou maior gravidade - as violências leves, como as palmadas, e as violências graves, como um soco) e podem se configurar como fenômenos qualitativamente distintos, podendo, por sua vez, apresentar mecanismos de produção também diferenciados (Afifi et al., 2017; Gershoff e Grogan-Kaylor, 2016).

A Organização Mundial da Saúde e a Sociedade Internacional para a Prevenção dos Abusos e das Negligências às Crianças (World Health Organization [WHO] \& International Society for Prevention of Child Abuse and Neglect [ISPCAN], 2006) propõem que o termo maus-tratos a crianças (child maltreatment) seja concebido como um conceito "guarda-chuva", 
abrangendo todas as formas de abusos e de negligências situadas no contexto de uma relação de responsabilidade, confiança e poder (com um cuidador/ responsável), com potencial de resultar em prejuízo para a sobrevivência da criança, para sua saúde, seu desenvolvimento ou sua dignidade. Estabelecem que os abusos físicos consistem em "uso intencional da força física contra a criança que resulta em - ou tem alta probabilidade de resultar em - prejuízo para a sobrevivência da criança, para a sua saúde, seu desenvolvimento ou sua dignidade". Isso inclui bater, surrar, chutar, chacoalhar, morder, estrangular, queimar/ escaldar, envenenar e sufocar, sendo que muitas dessas ações seriam infligidas com o objetivo de punir (WHO \& ISPCAN, 2006, p. 10). Nessa definição, a ação abusiva não precisa necessariamente causar lesão no corpo; pode ser apenas potencialmente perigosa, ameaçar o desenvolvimento e/ou ferir a dignidade da criança. Nessa linha, afirma-se que tanto a punição corporal quanto os abusos físicos são formas de violência física, pois remetem ao uso da força física sobre o corpo da criança/adolescente, tendo ambas as ações, portanto, a mesma qualificação.

Em síntese, há quem indique que apenas alguns comportamentos punitivos mais violentos configuram abuso (Ferguson, 2013; Larzelere, Gunnoe, \& Ferguson, 2018; Larzelere, Gunnoe, Ferguson, \& Roberts, 2019), e quem considere que qualquer forma de punição física é abusiva na medida em que fere a dignidade da criança, tomando como base as proposições da Convenção dos Direitos da Criança promulgada em 1989 (Durrant et al., 2019; Finkelhor, Turner, Wormuth, Vanderminden, \& Hamby, 2019; Organização dos Estados Americanos, 2009; Sege \& Amaya-Jackson, 2017; Smith, 2012).

Pesquisadores já tentaram identificar variáveis que pudessem discriminar um fenômeno de outro, todavia, até o momento, não obtiveram sucesso. A presença ou não de lesões (injury) é a variável mais frequentemente cogitada na distinção entre punição física/corporal e abuso físico. A validade desse critério tem sido, contudo, questionada por estudos empíricos que demonstram a incapacidade da variável "lesões" de discriminar o que seria castigo corporal do que seria abuso físico (Durrant, Trocmé, Fallon, Milne, \& Black, 2009). Em estudo recente com amostra nacionalmente representativa, no Canadá, Durrant et al. (2017) demonstram que em menos de um quarto das situações confirmadas como maus-tratos ou abusos pelo sistema de proteção infantil havia a presença de lesões físicas ou injúrias.

Assim, outros critérios são também cogitados na diferenciação entre castigo corporal e abuso físico: a natureza da intenção do responsável/cuidador (corrigir ou causar dor); o uso ou não de instrumentos (artifícios); a parte do corpo da criança na qual a punição incide. Essas variáveis parecem importantes, mas nem sempre discriminam fenômenos, impondo a necessidade de mais estudos (Durrant \& Enson, 2012; Durrant et al., 2017).

Longe do debate jurídico e acadêmico, na comunidade, percebe-se a existência de alguma distinção, em termos de aceitação, entre as inúmeras situações em que adultos (pais/responsáveis) usam força física, sobre os corpos das crianças/adolescentes (filhos/ filhas), suscetível de produzir sofrimento (dor). Nesse âmbito, ao mesmo tempo em que há uma ampla aceitação dos castigos físicos, há também o estabelecimento de alguns limites às ações parentais (dos responsáveis), levando em conta a existência de casos que não são notificados e são notificados ao sistema de proteção infantil, a despeito de todos os preceitos e indicativos legais e/ou científicos. Diante disso, parece importante perguntar se existem características passíveis de diferenciar grupos de pais/cuidadores cujas práticas/ações físicas são aceitas na comunidade (na medida em que não são notificadas) e não são aceitas na comunidade (na medida em que são notificadas). Assim, o presente estudo relata a investigação norteada pelo objetivo de verificar se grupos de pais/cuidadores sem e com histórico de notificação ao sistema de proteção por abuso se diferenciavam quanto às ações físicas sobre o corpo da criança, em termos de modalidades, frequência, partes do corpo da criança sobre as quais incidem e presença de sentimento de raiva no momento da punição, bem como quanto a variáveis psicossociais apontadas pela literatura científica como fatores de risco significativos para o abuso físico - como estresse, ansiedade, depressão, autoestima, estratégias de enfrentamento de problemas (coping), habilidades parentais e relacionamento com outros e com as crianças (Bérgamo \& Bazon, 2012; Ridings, Beasley, \& Silovsky, 2017; Rodriguez, Russa, \& Kircher, 2015).

\section{Método}

A abordagem adotada no estudo foi quantitativa, com delineamento transversal e comparativo. 
Os participantes foram 87 pais/cuidadores residentes em uma cidade de pequeno porte (aproximadamente 40 mil habitantes) na região de Ribeirão Preto - SP, que possuíam crianças e/ou adolescentes sob sua responsabilidade. Em função do objetivo da pesquisa, os participantes foram recrutados em dois diferentes contextos: 47 foram recrutados em equipamentos relacionados a programas comunitários e pára-escolares (locais em que as crianças permanecem no contra turno escolar), de modo a compor uma amostra de pais/cuidadores sem histórico de notificação (Grupo A); 40 foram recrutados no contexto de órgãos que compõem o sistema de proteção à infância, especificamente o Centro de Referência Especializado de Assistência Social (CREAS), a partir dos registros relativos à prática de maus-tratos, mais especificamente de abusos físicos, de modo a compor uma amostra de pais/cuidadores com histórico de notificação (Grupo B). É importante mencionar que, em princípio, 60 pais/responsáveis foram recrutados no contexto dos programas comunitários.
Contudo, 13 desses informaram não fazer uso de qualquer forma de punição corporal junto aos filhos. Assim, seus dados foram excluídos das análises e trabalhou-se com os dados de 47 pais/responsáveis.

A maior parte da amostra foi composta por indivíduos do sexo feminino (45 mulheres e 2 homens no Grupo A; 35 mulheres e 5 homens no Grupo B). A média de idade dos participantes nos dois grupos se mostrou equivalente, sendo de 37,5 anos ( $D P=12$ ) no Grupo A e de 34,3 anos (DP=9,4) no Grupo B $(\mathrm{p}=0,34)$. Em termos de escolaridade, os grupos também se mostraram equivalentes: no Grupo $\mathrm{A}$, a média de anos estudados foi de 6,8 (DP=3,3); no Grupo B, foi de $6,4(\mathrm{DP}=3,1 ; \mathrm{p}=0,76)$. No tocante ao nível socioeconômico, a Figura 1 sintetiza as informações oferecidas pelos participantes, segundo os parâmetros do Critério Brasil (Associação Brasileira de Empresas de Pesquisa [ABEP], 2014). Nota-se que, em ambos os grupos, as famílias se enquadram preponderantemente na classificação do estrato DE, sendo essa condição mais frequente no grupo B.

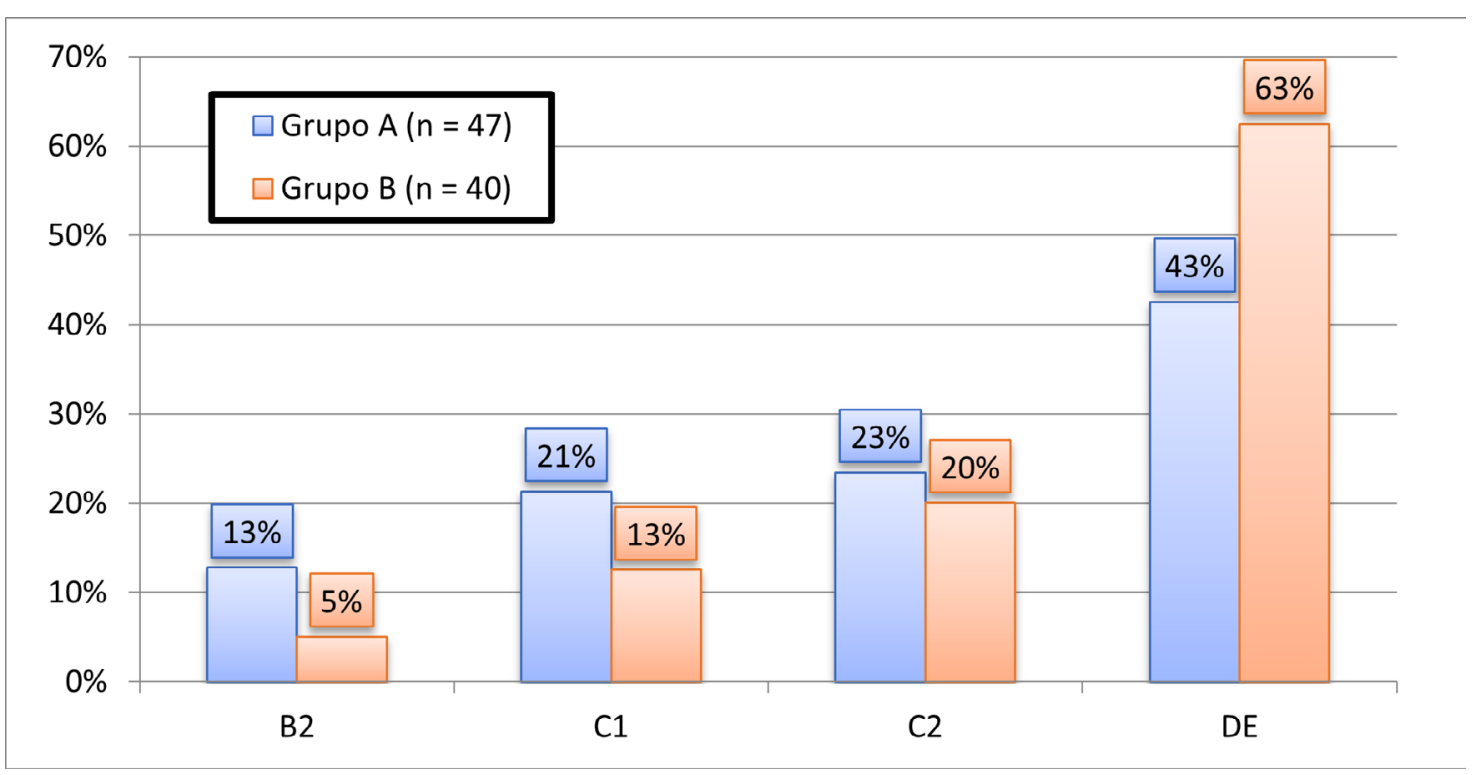

Figura 1

Classificação socioeconômica dos participantes dos grupos A e B de acordo com a Classificação Critério Brasil (ABEP, 2014).

Três instrumentos foram utilizados para coleta dos dados. O Questionário de Caracterização Sociodemográfica, originalmente proposto por Bringiotti (1999), tem por objetivo levantar informações que possibilitem caracterizar sociodemograficamente o(s) adulto(s) responsável(is) pelas crianças. Agregou-se a ele as questões inerentes ao Critério
Brasil, da Associação Brasileira de Empresas de Pesquisa, cujo objetivo é estimar o poder de consumo das famílias/domicílios, permitindo realizar classificação socioeconômica (ABEP, 2014).

O Roteiro de Entrevista Semi-Estruturada sobre Prática Parental, composto por 29 perguntas, foi elaborado pelas pesquisadoras com o objetivo de coletar 
informações sobre as práticas parentais, com especial atenção às de castigo/punição corporal, com vistas a caracterizá-las em termos de modalidade (modo de agir), frequência, motivações/razões para castigar/ punir fisicamente, situações e condição emocional ao aplicar a punição corporal e percepção sobre a eficácia da punição corporal.

O Inventário de Potencial de Maus-Tratos Infantil (Child Abuse Potential Inventory-CAP), elaborado por Joel Milner, em 1986, foi projetado para examinar pais/ cuidadores quanto ao risco de abuso físico infantil, tendo como base a aferição de determinadas características psicossociais. Os estudos de avaliação de suas qualidades psicométricas, no contexto de origem, ofereceram evidências das suas qualidades psicométricas (Milner, 1994). Estudos de validade simultânea relataram que as taxas de correta classificação de casos de abuso, por meio do escore na escala principal do CAP, variaram entre $80 \%$ e $90 \%$. Os de validade preditiva relataram que escores elevados em sua escala principal, indicando pais/cuidadores de alto risco, se mostraram forte e significativamente relacionados a casos posteriores de abuso físico infantil (Milner, 1994). O CAP já foi traduzido e adaptado em vários países, sendo amplamente utilizado na detecção do potencial de abuso infantil, bem como na avaliação da eficácia de intervenções na área. No Brasil, foi traduzido e adequado semanticamente para uso em pesquisas (Ávila de Mello et al., 2008). Rios, Williams, Schelini, Bazon e Piñon (2013) investigaram e obtiveram evidências de confiabilidade e de validade do Instrumento. Os coeficientes de consistência interna encontrados foram, respectivamente, 0,90 e 0,95 (Bérgamo, Pasian, Mello, \& Bazon, 2009; Rios et al., 2013). O constructo principal - o potencial de abuso - explicou $39,6 \%$ da variabilidade total em uma amostra estudada por Rios et al. (2013). O instrumento é composto por 160 afirmações às quais o participante responde optando por "Concordo" ou "Discordo". Os seus itens formam escalas. A escala clínica primária (abuso) pode ser dividida em seis escalas fatoriais: angústia, rigidez, infelicidade, problemas com a criança e consigo mesmo, problemas com a família, problemas com os outros. Outras duas escalas do instrumento são força do ego e solidão. A escala angústia (36 itens) identifica problemas no ajustamento pessoal do cuidador, solidão, depressão, preocupação, confusão, rejeição, baixa autoestima e raiva. A escala rigidez (14 itens) identifica estilo parental rígido, ou seja, cuidadores com crenças e expectativas rígidas quanto ao comportamento infantil. A escala infelicidade (11 itens) avalia a existência de uma polaridade de conteúdo felicidade versus infelicidade frente à vida. A escala problemas com a criança e consigo (6 itens) avalia a tendência do cuidador a perceber a criança e a si próprio de maneira negativa, com habilidades limitadas e com poucas competências. A escala problemas com a família (4 itens) identifica dificuldades no relacionamento com a parentela (família extensa). A escala problemas com os outros (6 itens) avalia se o cuidador considera as relações sociais difíceis. A escala solidão (15 itens) avalia a percepção do respondente quanto a suas possibilidades de ajuda e a escola força do ego (40 itens) avalia a percepção do cuidador sobre sua estabilidade emocional e seus sentimentos de adequação nas relações interpessoais. Por fim, o instrumento contém as escalas de detecção de distorções nas respostas: inconsistência interna, resposta ao acaso, desejabilidade social e mentira. As pontuações de cada item não são fixas e cada item pode compor mais de uma escala. Quanto mais alto o valor na escala de abuso, maior o potencial do cuidador em termos de risco de maus-tratos. A pontuação máxima nessa escala é 486. Os dados de normatização indicam que escores iguais ou maiores a 215 pontos já indicam risco de abuso infantil, em razão das características psicológicas e relacionais avaliadas.

A coleta de dados foi realizada individualmente, pela pesquisadora responsável pelo estudo, na residência dos participantes, por meio da aplicação dos instrumentos mencionados. A opção por ir até a residência dos participantes atendeu a exigências éticas, no sentido de os poupar de deslocamentos e eventuais gastos para atender à pesquisa, mas também funcionou como estratégia para estabelecer um contato de melhor qualidade, em um contexto não institucional, controlado pelo participante. Acredita-se que essa característica da coleta de dados tenha favorecido a obtenção de informações qualitativamente superiores.

Os dados coletados com os instrumentos padronizados (Critério Brasil e CAP) foram corrigidos de acordo com suas normas e os escores foram compilados em banco de dados criado para o estudo. Os dados coletados com o Questionário de caracterização sociodemográfica e a Entrevista semiestruturada sobre a prática parental foram tratados por meio de análise de conteúdo de modo que pudessem ser categorizados a partir de categorias pré-estabelecidas em função dos objetivos do estudo (Bardin, 2011), para, em seguida, 
também serem compilados no banco de dados. Cumpre frisar que essas análises visaram, em um primeiro momento, identificar as práticas mencionadas espontaneamente pelos pais/cuidadores investigados e classificá-las em termos de modalidade de punição/ castigo. Em um segundo momento, as categorias foram organizadas em ordem crescente em termos de risco potencial de dano/agravo físico, atribuindo-lhes, assim, um escore representativo nessa ordenação. A ordenação foi feita tendo como base apontamentos da literatura (Alexandre, Nadanovsky, Moraes, \& Reichenheim, 2010; Fréchette, Zoratti, \& Romano, 2015; Guterman, Lee, Lee, Waldfogel, \& Rathouz, 2009) e de dois profissionais consultados, com formação em medicina e especialidade em pediatria. A tabela 1 apresenta as categorias que sintetizam as práticas de punição mencionadas, ordenadas em função das ponderações feitas sobre o risco potencial de dano/agravo (das potencialmente menos danosas às potencialmente mais danosas).

\section{Tabela 1}

As modalidades de punição e suas respectivas pontuações na escala de gravidade.

\begin{tabular}{lc}
\hline \multicolumn{1}{c}{ Modalidade } & Pontuação \\
\hline Colocar de joelhos & 1 \\
Puxar o cabelo & 1 \\
Puxar a orelha & 1 \\
Dar beliscões & 1 \\
Amarrar & 2 \\
Dar palmadas & 2 \\
Dar chineladas & 3 \\
Dar croques & 4 \\
Chacoalhar & 5 \\
Empurrar & 5 \\
Atirar objetos & 6 \\
Dar chibatada & 7 \\
Dar socos & 7 \\
Dar paulada & 7 \\
Estrangular & 8 \\
Bater a cabeça contra a parede & 8 \\
\hline
\end{tabular}

Em seguida, os dados sobre as práticas também foram analisados de modo a identificar e a classificar as informações em termos de categorias concernentes à região principal do corpo da criançaladolescente sobre o qual incide a punição/castigo. As categorias, descritas na tabela 2, também foram ordenadas em termos de potencial de risco de agravos à saúde (do menor para o maior potencial de risco), segundo apontamentos da literatura e dos profissionais da pediatria consultados.

\section{Tabela 2}

Regiões do corpo da criança/adolescente sobre os quais incide a punição e as respectivas pontuações atribuídas, em termos de gravidade.

\begin{tabular}{cc}
\hline Região do corpo & Pontuação \\
\hline Abaixo da cintura & 1 \\
Mãos e braços & 2 \\
Tronco (costas ou peito) & 3 \\
Cabeça e face & 4 \\
\hline
\end{tabular}

As informações oferecidas pelos pais/cuidadores com relação à frequência com a qual recorreriam às práticas de punição corporal também foram consideradas. A frequência é um elemento importante, pois, se a punição corporal for muito frequente, mesmo que a modalidade da punição não seja tão gravosa, o risco de sofrimento físico e psicológico se eleva (Taillieu, Afifi, Mota, Keyes, \& Sareen, 2015). O dado da frequência foi classificado em uma das categorias pré-estabelecidas no roteiro de entrevista (diariamente; 2 ou 3 vezes por semana; uma vez por semana; uma vez por mês; menos de uma vez por mês).

Nas informações levantadas junto aos pais/ cuidadores investigados, também se buscou verificar a referência ao sentimento de raiva/irritação durante $o$ ato de punir/castigar, de forma a pontuá-lo. As ponderações na literatura indicam que a presença de sentimento de raiva ou de irritação durante o ato de punir/ castigar corporalmente caracteriza-o como uma descarga de emoções negativas, com mais risco de produzir dano/agravo físico e/ou psicológico à criança/ adolescente, destituindo a prática de qualquer valor instrumental (Martín, 2003; Plate et al., 2019).

Estabeleceu-se, assim, a possibilidade de aferir níveis de gravidade das práticas de castigo/punição corporal, pela combinação dessas quatro variáveis descritas: a) modalidade de punição; b) região do corpo da criança sobre o qual incidiria a punição; c) frequência da punição; ed) estadoemocionaldurante a punição. Os dados assim compilados foram tratados estatisticamente. A análise exploratória foi realizada a fim de organizar as informações e caracterizar os grupos estudados, em termos das médias e desvios-padrão para as variáveis quantitativas e em termos de 
frequência para as variáveis categóricas. Em seguida, os grupos foram comparados em relação às variáveis categóricas, empregando-se o teste de Qui-quadrado, e em relação às escalas do instrumento CAP, por meio do teste não-paramétrico U de Mann-Whitney. O projeto da pesquisa foi analisado e aprovado pelo Comitê de Ética e Pesquisa com Seres Humanos da Faculdade de Filosofia, Ciências e Letras de Ribeirão Preto da Universidade de São Paulo (Protocolo de aprovação CAEE n. 368416614.8.0000.5407).

\section{Resultados}

A tabela 3 apresenta uma síntese dos dados no tocante às modalidades de punição, às regiões do corpo sobre as quais incide a punição, à frequência da punição e à presença de sentimento de raival irritação por ocasião da punição, nos dois grupos e a comparação entre eles.

Depreende-se da tabela que os grupos A e B não se diferenciaram apenas quanto à presença de sentimento de raiva durante o ato de punir. Nas outras variáveis, o grupo B apresentou médias mais elevadas; os pais/responsáveis nesse grupo utilizariam modalidades de punição corporal potencialmente mais gravosas, em regiões do corpo mais sensíveis/ frágeis, com maior frequência. A Tabela 4 sintetiza os resultados relativos ao Inventário de Potencial de Maus-tratos Infantis (CAP).

Tabela 3

Indicadores de gravidade das práticas de punição/castigo corporal nos Grupos A e B e comparação entre os grupos.

\begin{tabular}{|c|c|c|c|c|c|c|c|}
\hline & \multicolumn{2}{|c|}{ Grupo A $(n=47)$} & \multicolumn{2}{|c|}{ Grupo B $(n=40)$} & \multirow[b]{2}{*}{$\mathbf{U}$} & \multirow[b]{2}{*}{$\mathbf{p}$} & \\
\hline & $\mathbf{M}$ & DP & $\mathbf{M}$ & DP & & & \\
\hline Modalidades de punição' & 7,8 & 5,9 & 17,1 & 7,8 & 285 & 0,00 & $* *$ \\
\hline Região do corpo ${ }^{1}$ & 3,2 & 2,6 & 7,3 & 4,0 & 363,5 & 0,00 & $* *$ \\
\hline \multirow[t]{2}{*}{ Frequência da punição } & 1,6 & 0,8 & 2,3 & 0,9 & 549 & 0,00 & $* *$ \\
\hline & $\mathbf{n}$ & $\%$ & $\mathbf{n}$ & $\%$ & $X^{2}$ & $\mathbf{p}$ & \\
\hline Presença raiva $^{2}$ & 39 & $83 \%$ & 34 & $85 \%$ & 0,1 & 0,80 & \\
\hline
\end{tabular}

1. Teste de Mann-Whitney

2. Teste de Qui-quadrado

** Estatisticamente significante ao nível de 0,01

Tabela 4

Escores médios nas escalas do CAP e comparação entre os grupos A e B.

\begin{tabular}{cccccccc}
\hline & \multicolumn{2}{c}{ Grupo A } & \multicolumn{2}{c}{ Grupo B } & \\
\cline { 2 - 6 } & M & DP & M & DP & U & p & \\
\hline Abuso & 209,0 & 105,7 & 282,2 & 92,0 & 566 & 0,00 & $* *$ \\
Angústia & 121,1 & 73,5 & 156,8 & 69,8 & 676 & 0,02 & $*$ \\
Rigidez & 30,8 & 14,6 & 46,7 & 11,4 & 363 & 0,00 & $* *$ \\
Infelicidade & 22,9 & 15,5 & 33,2 & 16,4 & 590 & 0,00 & $* *$ \\
Prob. consigo/cça & 4,0 & 5,5 & 6,6 & 6,9 & 701,5 & 0,04 & $*$ \\
Prob. família & 15,1 & 13,2 & 20,7 & 14,8 & 747,5 & 0,10 & \\
Prob. outros & 14,4 & 7,9 & 18,4 & 6,7 & 634 & 0,01 & $* *$ \\
Força do ego & 20,6 & 10,0 & 14,0 & 9,3 & 581 & 0,00 & $*$ \\
Solidão & 8,3 & 4,4 & 10,1 & 4,4 & 698,5 & 0,04 & $*$ \\
\hline
\end{tabular}

**Estatisticamente significante ao nível de 0,01

* Estatisticamente significante ao nível de 0,05 
Notam-se diferenças significativas entre os grupos na escala principal, a de abuso, e nas suas subescalas, angústia, rigidez, infelicidade, problemas consigo e com acriança, problemas comosoutros, assim como em força do ego e solidão. Na escala força do ego, o grupo A apresentou escore médio maior que o grupo $\mathrm{B}$, lembrando que ela é uma escala invertida (ou seja, quanto maior a média, maior a força do ego). Cumpre mencionar, quanto às escalas de validade (mentira, acaso e inconsistência), que os valores médios foram adequados, tomando como referência os padrões normativos americanos (Milner, 1994) e os padrões normativos brasileiros, produzidos especificamente para essas escalas por Rios (2010), ou seja, os valores médios nessas escalas indicaram a validade das respostas nos dois grupos estudados (em termos médios).

\section{Discussão}

No presente estudo, adotou-se o posicionamento teórico de que variadas formas de punição corporal/ castigo físico constituem-se em maus-tratos físicos, uma das modalidades de violência doméstica contra crianças e adolescentes, independentemente do fato de causar ou não lesões, uma vez que, qualquer ação que seja coloca a criança/adolescente em uma posição de objeto e fere sua dignidade. Assim, coaduna-se com a definição ampla proposta pela Organização Mundial da Saúde e pela Sociedade Internacional para a Prevenção dos Abusos e Negligências em Crianças (WHO \& ISPCAN, 2006). Pressupôs-se, contudo, a existência de diferentes níveis de gravidade do problema, devendo-se entender os maus-tratos físicos como um fenômeno heterogêneo, seja em termos do comportamento manifesto (natureza e frequência), seja em termos dos fatores de risco associados (Gershoff \& Grogan-Kaylor, 2016; Larrivée, Tourigny, \& Bouchard, 2007). No que tange ao comportamento de punir fisicamente, os resultados apontam que os grupos investigados, formados em função do fato de terem ou não sido notificados ao sistema de proteção infantil por abuso físico, se distinguiram em relação a todas as variáveis que compuseram o constructo de gravidade, à exceção da presença do sentimento de raiva/irritação. Dito de outro modo, o grupo de pais/ responsáveis notificados utilizariam modalidades de punição com maior potencial de provocar dano físico, sendo que essas incidiram mais em regiões do corpo da criançaladolescente mais vulneráveis, ou seja, em regiões acima da cintura (incluindo a cabeça), e foram mais frequentes (em média, duas vezes mais).
Esses resultados denotam a existência de práticas de punição corporal diferentes, em termos de gravidade, e também evidenciam a regulação comunitária dos casos, considerando-se a reação aos casos mais graves - suscetíveis de impactar mais prontamente e, talvez, mais severamente, o desenvolvimento infantilpor meio da notificação, assim como a tolerância/ aceitação de casos menos graves. Isso significa que, apesar de existirem limites socialmente impostos àquilo que se faz com os "corpos das crianças e dos adolescentes", há também uma ampla aceitação dos castigos físicos/corporais como estratégia de disciplina/educação infantil, ou seja, os comportamentos parentais de punição física considerados mais leves não são vistos socialmente como violência contra criança, o que se configura como um grande desafio para os gestores e profissionais que atuam no âmbito da saúde pública e da proteção social (Durrant et al., 2019; Organização Mundial da Saúde [OMS], 2016; WHO \& ISPCAN, 2006). Para além da questão do subdimensionamento da violência que decorre dessa prática social, que concorre para políticas públicas equivocadas no tocante à magnitude do problema em foco, tem-se o fato de que a ampla aceitação das punições físicas/castigos corporais menos graves endossa e fomenta uma cultura de tolerância das práticas violentas, ainda que imbuídas de objetivos educativos/disciplinares.

A propósito disso, deve-se considerar que o caráter educativo/disciplinar que a prática de punição corporal poderia, em certa medida, assumir, segundo alguns teóricos, pressupõe o controle emocional dos pais/adultos durante o ato (Ferguson, 2013; Larzelere et al., 2018; Larzelere et al., 2019). Nesse tocante, os resultados obtidos mostram que, nos dois grupos investigados, a maioria indicou o sentimento de irritação ou raiva no momento da punição corporal, não se diferenciando nesse quesito. Assim, mesmo as punições corporais menos graves não seriam defensáveis, pois não alcançam uma das condições para que pudesse ser funcional, em termos educativos/disciplinares. Os resultados obtidos, em consonância com o que argumenta Martín (2003), remetem à questão de se e quando a punição corporal pode efetivamente ser uma estratégia educativa/ disciplinar, quando, em efeito, representa mais propriamente uma "descarga emocional", função de uma necessidade dos adultos/responsáveis de alívio de tensão, frustração e raiva. 
Enquanto ação que expressa sentimentos negativos da parte do adulto, é mais fácil entender por que o castigo corporal se constitui em fator de risco aos abusos físicos, já que, na ausência de práticas parentais efetivamente educativas, os comportamentos infantis/adolescentes podem se complexificar e, com isso, a reatividade negativa do adulto frente a eles também risca se intensificar. Quando os pais/ responsáveis se engajam no comportamento de punir fisicamente seus filhos, normalmente não têm a intenção de machucá-los; todavia, alguns pais/cuidadores, imbuídos da crença de que têm o direito de punir fisicamente os filhos e de que esse comportamento se justifica, na presença de fatores de risco psicossociais aos maus-tratos, frequentemente agravam a punição aplicada (Durrant et al., 2017). Gershoff e GroganKaylor (2016) apontam, com base em metanálise, que a prática da punição corporal é o principal fator de risco para o abuso físico infantil.

Esse limite implementado espontaneamente na comunidade não se presta, portanto, para indicar o que é e o que não é maus-tratos. Esse limite, talvez, seja indicativo dos diferentes níveis de intervenção de prevenção e de enfrentamento que precisam ser concebidos e efetivados por meio de programas da saúde e socioassistenciais, conforme prevê a lei. O ECA (Lei n. 8.069, 1990) prevê em seu artigo 18-B que os pais, os integrantes da família ampliada, os responsáveis, os agentes públicos ou qualquer pessoa encarregada de cuidar de crianças e adolescentes que utilizem castigos físicos estão sujeitos, sem prejuízo de outras sanções cabíveis, às seguintes medidas: a) encaminhamento a programa oficial ou comunitário de proteção à família; b) encaminhamento a tratamento psicológico ou psiquiátrico; c) encaminhamento a cursos ou programas de orientação; d) obrigação de encaminhar a criança a tratamento especializado; e e) advertência. As medidas preconizadas na lei, embora sem clareza técnica, revelam o reconhecimento, por parte do Estado, da necessidade de intervenções diferenciadas. Nesse âmbito, é necessário que os agentes que irão determinar quais medidas serão cumpridas possam ter critérios claros para identificar as necessidades de cada família/indivíduo. Do contrário, corre-se o risco de que as famílias sejam encaminhadas a intervenções ineficazes ou desnecessárias, o que, além de representar um desperdício de recursos financeiros e humanos, pode concorrer para a permanência da situação na qual os maus-tratos são originados.
Alinhando-se a esses apontamentos sobre a necessidade de conceber e implementar diferentes níveis de intervenção, verificou-se que os participantes das famílias notificadas ao sistema de proteção infantil se diferenciaram daqueles das famílias sem esse histórico quanto às características psicossociais. Os resultados da comparação dos grupos no Inventário de Potencial de Abuso Infantil (CAP) evidenciam diferenças significativas na escala principal, a de abuso, e nas subescalas angústia, infelicidade, rigidez, problemas consigo e com a criança, problemas com os outros e solidão, denotando que os pais/ responsáveis notificados apresentariam em maior frequência e intensidade as dificuldades mensuradas por essas escalas. Eles apresentariam maior desconforto psicológico, evidenciado por sentimentos de baixa autoestima, tristeza, confusão e ansiedade, corroborando achados de trabalhos científicos que caracterizam pais/cuidadores implicados em situações de violência (Ridings et al., 2017; Rodriguez et al., 2015).

Os escores na escala de abuso do presente estudo se mostraram semelhantes aos de outros trabalhos brasileiros que utilizaram o CAP, sobretudo no que se refere aos participantes notificados. O escore médio de 282,2 nele se equipara ao dos grupos clínicos, ou seja, aos grupos de outros estudos em que há presença de abuso físico comprovado: 258,4 (Ávila de Mello, 2008), 265,5 (Patrian, 2009) e 223,9 (Bérgamo \& Bazon, 2012). Quanto a este aspecto, merece destaque, também, o fato de o grupo de participantes não notificados ao sistema de proteção apresentar escore médio de 209, índice bem mais elevado que o identificado nos grupos controles dos outros estudos citados: 149,6 (Ávila de Mello, 2008), 103,4 (Patrian, 2009) e 166,9 (Bérgamo \& Bazon, 2012). No presente trabalho, conforme apresentado, o escore de 209 é mais próximo ao escore relativo à nota de corte indicada pelo manual do CAP, em seu país de origem, como indicativa de risco de abuso infantil (215 pontos). Pode-se cogitar, mediante esse resultado, que os pais/responsáveis não notificados por abuso físico, ainda que recorressem a práticas de punição corporal menos graves (com menor risco de ocasionar agravos ao desenvolvimento infantil), apresentariam também fragilidades psicológicas atinentes aos abusos físicos, dado que fortalece a importância de haver ações de prevenção primária e secundária concernentes à punição corporal, de forma geral. 
Quanto aos sentimentos de angústia e de infelicidade parental, nota-se que o grupo notificado apresentou escores mais elevados em ambas as subescalas, o que aponta para a preponderância de um padrão de sentimentos marcado por mal-estar psicológico, no qual se evidenciam sensações de frustração, tristeza, solidão, depressão, preocupação, confusão e infelicidade quanto à vida em geral e aos relacionamentos. Segundo a literatura, esses sentimentos negativos interferem na capacidade do cuidador/responsável de se voltar para a criança, no sentido de permanecer sensível e disponível, pois o mal-estar psicológico vivenciado pode fazer com que ele fique centrado em seus próprios sentimentos e necessidades, dificultando a identificação e a compreensão adequada dos comportamentos infantis (Bérgamo \& Bazon, 2012; Rodriguez, Baker, $\mathrm{Pu}, \&$ Tucker, 2017; Venta, Velez, \& Lau, 2016).

Os grupos também se mostraram significativamente diferentes na subescala rigidez. Os pais/ responsáveis notificados pontuaram mais alto. O padrão de funcionamento psicológico aferido pelos itens dessa escala tem sido apontado pela literatura internacional como central à produção do abuso físico infantil. Esse padrão se refere a um funcionamento cognitivo com pouca flexibilidade para avaliar as situações de maneira particularizada, ou seja, considerando e integrando as características específicas de cada situação (Azar et al., 2016; Milner, 2003). Os pais que apresentam problemas no processamento da informação social, por conta de tal rigidez, tendem a atribuir intenções hostis à criança, acreditando que ela se comporta mal de maneira proposital porque tem a intenção de provocar. Desse modo, avaliam o comportamento da criança como mais errado, mais grave e mais condenável. Ademais, acreditam que os comportamentos infantis que consideram inadequados ocorrem em função de características pessoais, estáveis e globais, da criança, ou seja, os indivíduos com marcada rigidez têm grande dificuldade em considerar as variáveis situacionais, o processo de desenvolvimento infantil e as transformações decorrentes dele. Desse modo, quanto maior a rigidez, maior a probabilidade de que a ação "educativa/ disciplinar" seja mais severa e que resulte em punições físicas mais graves (Azar et al., 2016; Beckerman, van Berkel, Mesman, \& Alink, 2017).

É importante ressaltar que os grupos também se mostraram significativamente diferentes na escala força do ego, a única escala positiva do CAP, com os pais/responsáveis notificados por abuso físico pontuando mais baixo. À semelhança do que foi explicitado quanto aos sentimentos de angústia e infelicidade, a sensação de vulnerabilidade emocional, expressa pelos menores índices na escala força do ego, pode se configurar como fator mediador dos processos cognitivos parentais, favorecendo não apenas menor disponibilidade afetiva para lidar com as demandas dos filhos, mas também distorções nas percepções, nas expectativas e nas interpretações do comportamento infantil (Azar et al., 2016; Milner, 2003). Pondera-se que os sentimentos de angústia e de infelicidade e a menor força do ego propiciam um pano de fundo emocional e cognitivo desfavorável à adequada assunção das tarefas parentais, de cuidado e de socialização.

$\mathrm{Na}$ esteira da maior fragilidade psicológica/ emocional e da maior rigidez cognitiva e comportamental apresentada pelos pais/cuidadores notificados, era esperado que tivessem índices significativamente maiores nas escalas problemas com a criança e consigo e problemas com os outros, o que efetivamente foi constatado. Em uma perspectiva dinâmica, o mal-estar psicológico, associado à inadequação nas percepções e nas expectativas, concorreria para a produção de problemas no relacionamento com as crianças e, também, com as outras pessoas do entorno social. Esses problemas relacionais, por seu turno, retroalimentariam as dificuldades psicológicas e emocionais ressentidas pelos indivíduos que apresentam tais características. Bérgamo e Bazon (2012) sublinham que a percepção dos relacionamentos sociais como um problema pode gerar maior isolamento socioemocional e potencializar a solidão e o estresse vivenciados.

É importante sublinhar que os grupos não se diferenciaram significativamente na escala problemas com a família, resultado que merece atenção. Em outros estudos brasileiros que utilizaram o CAP, nota-se que os escores da escala problemas com a família foram menores tanto para os grupos controle quanto para os grupos clínicos, quando comparados aos escores do presente trabalho. Ávila de Mello (2008) encontrou escore de 7,4 para grupo controle e de 17,2 para grupo clínico; Patrian (2009) encontrou escore de 1,6 para grupo controle e de 9,35 para grupo clínico; Bérgamo e Bazon (2012) encontraram escore de 9,0 para grupo controle e de 12,63 para 
grupo clínico. No presente trabalho, a pontuação do grupo controle foi de 15,1 e a do grupo clínico foi de 20,7. Assim, pode-se afirmar que as pontuações da escala de problemas com a família são mais elevadas em ambos os grupos investigados no presente estudo, apontando, possivelmente, para a existência de problemas no apoio social recebido/percebido por ambos junto à parentela.

Finalmente, cabe tecer considerações a respeito da escala solidão, na qual também houve diferenças significativas entre os grupos. Os itens dessa escala, além de representarem aspectos psicológicos do indivíduo, como sentimentos de desconfiança em relação às pessoas e sensação de rejeição, abordam o apoio, ou melhor, a percepção de falta de apoio e o sentimento de isolamento social. Assim, depreende-se que os participantes notificados no sistema de proteção infantil, por terem obtido maior média, sentir-se-iam mais desconfortáveis em seus relacionamentos sociais, percebendo-se, ainda, como pouco apoiados e como isolados socialmente, reiterando a avaliação em escalas como problemas com a criança e consigo e problemas com os outros. As subjetividades dos dois grupos, assim apreendidas, permitem vislumbrar que os pais/responsáveis investigados engajados em práticas de punição corporal mais graves apresentariam, também, muito mais problemas emocionais, cognitivos e relacionais que aqueles engajados em práticas menos graves. Assim, novamente tem-se a indicação da necessidade de conceber e de implementar programas de intervenção (seja em prevenção, seja em tratamento) diferenciados.

Em síntese, os resultados do presente trabalho denotaram que o castigo físico ainda é um recurso amplamente utilizado para disciplinar as crianças, mas também apontaram para a existência de diferenças importantes tanto no comportamento manifesto quanto nas variáveis psicossociais associadas ao uso de punição corporal contra criança/adolescente em grupos de pais/responsáveis em uma mesma comunidade. Pais/responsáveis sem o histórico de notificação por abuso físico apresentaram práticas com menor potencial de dano e menos problemas psicossociais quando comparados a pais/responsáveis que foram notificados ao sistema de proteção à infância. Estes apresentaram comportamentos potencialmente mais danosos, maior fragilidade emocional, maior rigidez cognitiva e instabilidade emocional e mais sentimentos de solidão e isolamento social.
Pode-se supor que intervenções universais, em nível prevenção primária, de cunho educativo com divulgação de informações científicas a respeito da ineficácia e das consequências negativas para as crianças submetidas ao castigo físico, associadas à divulgação de outras formas de disciplina assertiva - podem ser suficientes para minimizar, ou até mesmo extinguir, o uso dos castigos físicos junto a um grande conjunto de pais/responsáveis da comunidade, menos exposto a fatores de risco psicossociais específicos. Junto aos pais/responsáveis identificados como mais vulneráveis, que lançam mão de práticas de punição física mais graves, ao que tudo indica, seriam necessárias intervenções mais sofisticadas, em nível de programas/serviços de prevenção secundária ou mesmo terciária, envolvendo diversos setores da rede de proteção, talvez de natureza mais clínica (para além de educativa), visando ao fortalecimento de recursos pessoais e de vínculos familiares e comunitários, dentre outros aspectos relevantes.

\section{Considerações finais}

O presente estudo teve por objetivo verificar se grupos de pais/responsáveis sem e com histórico de notificação ao sistema de proteção por abuso físico se diferenciavam quanto às ações físicas sobre o corpo da criança, em termos de gravidade, levando em conta modalidade, frequência, partes do corpo da criança sobre as quais incide e presença de sentimento de raiva parental no momento da punição. Ademais, buscou-se verificar diferenças entre os grupos no tocante a variáveis psicossociais apontadas pela literatura como fatores de risco significativos para o abuso físico. Os resultados indicaram que, no grupo de pais/responsáveis notificados, as punições corporais seriam mais graves, exceto pela presença de sentimento de raiva, aspecto que caracterizaria a prática disciplinar em ambos os grupos. Tais diferenças no plano do comportamento de punir fisicamente se mostraram associadas a diferenças nos planos da maior parte das variáveis psicossociais avaliadas. O grupo de pais notificados apresentaria maior fragilidade emocional com maior prevalência de sintomas de mal-estar psicológico, como sentimentos de tristeza, preocupação, angústia, solidão, infelicidade e menor sensação de estabilidade e adequação emocional. O funcionamento cognitivo desses pais seria marcado por maior rigidez, importante fator de risco para o abuso físico. Tanto a fragilidade emocional 
quanto o funcionamento cognitivo marcado pela pouca flexibilidade prejudicam a capacidade dos cuidadores de interagirem com as crianças/adolescentes de maneira apropriada.

Em conjunto, todos os resultados reiteram a ideia de que todas as formas de punição corporal são maus-tratos físicos (expressões de violência contra a infância e a adolescência), mas que esses se constituem em um fenômeno heterogêneo, seja em termos de gravidade dos atos, seja em termos de fatores associados, o que precisa ser considerado na proposição de programas de prevenção e de tratamento. Vale ressaltar que o sentimento de raiva no ato da punição corporal, em ambos os grupos, ofereceu evidência para que se questione tanto os cientistas que defendem que essa prática pode, em certa medida, ser "funcional", quanto os legisladores brasileiros, mediante a existência de brechas nas leis, como a criada pelo Código Civil, que indica a possibilidade do uso do castigo "moderado".

Obviamente os resultados do presente trabalho podem ser ponderados frente a alguns limites metodológicos. Um deles refere-se ao fato de se ter buscado aferir o comportamento de punição por meio de uma abordagem de autorrelato. Para além da diferença entre "o que se faz" e "o que se fala sobre o que se faz", inerente a qualquer temática, tem-se o fato de a temática abordada ser delicada e poder ter gerado respostas enviesadas pela "desejabilidade social". Assim, pondera-se sobre uma possível subestimação nos dados quanto à frequência e aos tipos de castigos físicos relatados. A respeito disso, ressalta-se também o fato de se ter lidado com as formas de punição física relatadas espontaneamente, o que talvez tenha gerado perda de informação relacionada a formas menos usuais, como privar a criança de alimento. Na mesma direção, privilegiou-se avaliar a gravidade da punição corporal por meio da aferição de algumas variáveis, e se preteriu outras igualmente relevantes, como a quantidade de golpes deferidos. De igual modo, no tocante aos fatores de risco, privilegiou-se variáveis psicológicas relacionadas aos pais/cuidadores, deixando de lado variáveis ambientais/contextuais relevantes para a compreensão do fenômeno e para as intervenções na área. Ademais, tem-se que o delineamento transversal adotado não permite a avaliação de transformações nas práticas e, tampouco, no funcionamento psicológico dos investigados, aspecto central quando se estuda questões relacionadas ao desenvolvimento humano. Essas limitações podem, todavia, ser superadas com o empreendimento de novas investigações científicas, inclusive com vistas a ampliar e aprofundar o conhecimento relativo ao fenômeno da punição corporal infantil, fenômeno tão prevalente em nossa sociedade.

\section{Referências}

Afifi, T. O., Ford, D., Gershoff, E., Merrick, M., Grogan-Kaylor, A., Ports, K. A., MacMillan, H. L., Holden, G. W., Taylor, C. A., Lee, S. J., \& Bennett, R. P. (2017). Spanking and adult mental health impairment: The case for the designation of spanking as an adverse childhood experience. Child Abuse and Neglect, 71, 24-31. https://doi.org/ 10.1016/j.chiabu.2017.01.014

Alexandre, G. C., Nadanovsky, P., Moraes, C. L., \& Reichenheim, M. (2010). The presence of a stepfather and child physical abuse, as reported by a sample of Brazilian mothers in Rio de Janeiro. Child Abuse \& Neglect, 34(12), 959-966. https:// doi.org/10.1016/j.chiabu.2010.06.005

Associação Brasileira de Empresas de Pesquisa. (2014). Critério de Classificação Econômica Brasil. http://www.abep.org/ criterio-brasil

Ávila de Mello, I. L. M. (2008). Negligência de crianças: Contribuições para a avaliação de fatores de risco psicossociais [Dissertação de mestrado]. Universidade de São Paulo.

Ávila de Mello, I. L. M., Bérgamo, L. P. D., Piñon, E. A., Rios, K. S. A., Williams, L. C. A., \& Bazon, M. R. (2008). Inventário de Potencial de Abuso Infantil - CAP: Versão em Português. Universidade Federal de São Carlos; Universidade de São Paulo.

Azar, S. T., Miller, E. A., McGuier, D. J., Stevenson, M. T., O’Donnell, E., Olsen, N., \& Spence, N. (2016). Maternal social information processing and the frequency and severity of mother-perpetrated physical abuse. Child Maltreatment, 21(4), 308-316. https://doi.org/10.1177/1077559516668047

Bardin, L. (2011). Análise de Conteúdo (4a ed.). Setenta. 
Beckerman, M., van Berkel, S. R., Mesman, J., \& Alink, L. R. A. (2017). The role of negative parental attributions in the associations between daily stressors, maltreatment history and harsh and abusive discipline. Child Abuse and Neglect, 64, 109-116. https://doi.org/10.1016/j.chiabu.2016.12.015

Bérgamo, L. P. D., \& Bazon, M. R. (2012). Abuso infantil: Avaliando fatores de risco psicológicos em cuidadores notificados. Psicologia: Reflexão e Crítica, 25(2), 256-264. https://doi.org/10.1590/S0102-79722012000200007

Bérgamo, L. P. D., Pasian, S. R., Mello, I. L. M. A., \& Bazon, M. R. (2009). O Inventário de Potencial de Maus-Tratos Infantil: Estudo de precisão e validade. Avaliação Psicológica, 8(3), 425-435. http://pepsic.bvsalud.org/pdf/avp/ v8n3/v8n3a14.pdf

Bringiotti, M. I. (1999). Maltrato infantil: Factores de riesgo para el maltrato físico en La población infantil que concurre a las escuelas dependientes del Gobierno de la Ciudad de Buenos Aires. Niño y Dávila.

Constituição da República Federativa do Brasil. (1988). Diário Oficial da União de 05 de outubro de 1988.

Dias, M. B. (2014, 30 de junho). Lei da Palmada? Lei Menino Bernardo? https://ibdfam.org.br/artigos/965/ Lei+da+Palmada\%3F+Lei+Menino+Bernardo\%3F

Durrant, J., \& Ensom, R. (2012). Physical punishment of children: Lessons from 20 years of research. Canadian Medical Association Journal, 184(12), 1373-1377. https://doi.org/10.1503/cmaj.101314

Durrant, J. E., Fallon, B., Lefebvre, R., \& Allan, K. (2017). Defining reasonable force: Does it advance child protection? Child Abuse and Neglect, 71, 32-43. https://doi.org/10.1016/j.chiabu.2017.02.018

Durrant, J. E., Stewart-Tufescu, A., Afifi, T. O. (2019). Recognizing the child's right to protection from physical violence: An update on progress and a call to action. Child Abuse and Neglect, 110(pt. 1). https://doi.org/10.1016/ j.chiabu.2019.104297

Durrant, J. E., Trocmé, N., Fallon, B., Milne, C., \& Black, T. (2009). Protection of children from physical maltreatment in Canada: An evaluation of the Supreme Court's definition of reasonable force. Journal of Aggression, Maltreatment and Trauma, 18(1), 64-87. https://doi.org/10.1080/10926770802610640

Ferguson, C. J. (2013). Spanking, corporal punishment and negative long-term outcomes: A meta-analytic review of longitudinal studies. Clinical Psychology Review, 33(1), 196-208. https://doi.org/10.1016/j.cpr.2012.11.002

Finkelhor, D., Turner, H., Wormuth, B. K., Vanderminden, J., \& Hamby, S. (2019). Corporal Punishment: Current Rates from a National Survey. Journal of Child and Family Studies, 28(7), 1991-1997. https://doi.org/10.1007/ s10826-019-01426-4

Fréchette, S.; Zoratti, M., \& Romano, E. (2015). What is the link between corporal punishment and physical abuse? Journal of Family Violence, 30(2), 135-148. https://doi.org/10.1007/s10896-014-9663-9

Gershoff, E. T., \& Grogan-Kaylor, A. (2016). Spanking and child outcomes: Old controversies and new meta-analyses. Journal of Family Psychology, 30(4), 453-469. https:// doi.org/10.1037/fam0000191

Guterman, N. B., Lee, Y., Lee, S. J., Waldfogel, J., \& Rathouz, P. J. (2009). Fathers and maternal risk for physical child abuse. Child Maltreatment, 14(3), 277-290. https://doi.org/10.1177/1077559509337893

Larrivée, M-C, Tourigny, M., \& Bouchard, C. (2007). Child physical abuse with and without other forms of maltreatment: Dysfunctionality versus dysnormality. Child Maltreatment, 12(4), 303-313. https://doi.org/ $10.1177 / 1077559507305832$

Larzelere, R. E., Gunnoe, M. L., \& Ferguson, C. J. (2018). Improving causal inferences in meta-analyses of longitudinal studies: Spanking as an illustration. Child Development, 89(6), 2038-2050. https://doi.org/10.1111/cdev.13097

Larzelere, R. E., Gunnoe, M. L., Ferguson, C. J., \& Roberts, M. W. (2019). The insufficiency of the evidence used to categorically oppose spanking and its implications for families and psychological science: Comment on Gershoff et al. (2018). American Psychologist, 74(4), 497-499. https://doi.org/10.1037/amp0000461

Lei n. 8.069. (1990). Estatuto da criança e do adolescente. Diário Oficial da União de 13 de julho de 1990.

Lei n. 10.406/2002. (2002). Código Civil. Diário Oficial da União de 10 de janeiro de 2002.

Lei n. 13.010/2014. (2014). Lei Menino Bernardo. Diário Oficial da União de 27 de junho de 2014, retificado em 07 de julho de 2014. 
Milner, J. S. (1994). Assessing physical child abuse risk: The child abuse potential inventory. Child Psychology Review, 14(6), 547-583. https://doi.org/10.1016/0272-7358(94)90017-5

Milner, J. S. (2003). Social information processing in high-risk and physically abusive parents. Child Abuse and Neglect, 27(1), 7-20. https:// doi.org/10.1016/S0145-2134(02)00506-9

Martín, M. M. B. (2003). Las acciones punitivas físicas: Una perspectiva intercultural. Estudios Pedagógicos, 29, 85-95. https://doi.org/10.4067/S0718-07052003000100006

Organização dos Estados Americanos. (2009). Relatório sobre castigo corporal e os direitos humanos das crianças e adolescentes: Promovendo a defesa e o respeito dos direitos humanos das crianças e adolescentes nas Américas. http://www.cidh.org/pdf\%20files/castigo\%20corporal\%20portugues.pdf

Organização Mundial da Saúde. (2016). INSPIRE: Sete Estratégias para Pôr Fim à Violência Contra Crianças. https://apps.who.int/iris/bitstream/handle/10665/207717/9789241565356-por.pdf?ua=1

Patrian, A. C. A. (2009). Validade de critério para o Inventário de Potencial de Abuso Infantil - CAP [Trabalho de Conclusão de Curso]. Universidade Federal de São Carlos.

Plate, R. C., Bloomberg, Z., Bolt, D. M., Bechner, A. M., Roeber, B. J., \& Pollak, S. D. (2019). Abused children experience high anger exposure. Frontiers in Psychology, 10, 440. https://doi.org/10.3389/fpsyg.2019.00440

Ribeiro, F. B. (2016). A proibição legal de castigos físicos na infância: Alguns contrastes entre Brasil, Uruguai e França. Desidades, 10(4), 19-29.

Ridings, L. E., Beasley, L. O., \& Silovsky, J. F. (2017). Consideration of risk and protective factors for families at risk for child maltreatment: An intervention approach. Journal of Family Violence, 32(2), 179-188. https://doi.org/ 10.1007/s10896-016-9826-y

Rios, K. S. A. (2010). Inventário de Potencial de Abuso Infantil (CAP): Adaptação Transcultural, Fidedignidade e Validade para o Brasil [Tese de doutorado]. Universidade Federal de São Carlos.

Rios, K. S. A., Williams, L. C. A., Schelini, P. W., Bazon, M. R., \& Piñon, E. A. (2013). Inventário de Potencial de Abuso Infantil - CAP: Evidências de validade e precisão. Avaliação Psicológica, 12(1), 51-60.

Rodrigues, A., \& Tomé, P. I. (2014). Lei da Palmada não proíbe palmada, dizem advogados. Folha de S.Paulo. http://wwwl.folha.uol.com.br/cotidiano/2014/06/1465898-lei-da-palmada-nao-proibe-palmada-dizemadvogados.shtml

Rodriguez, C. M., Baker, L. R., Pu, D. F., \& Tucker, M. C. (2017). Predicting parent-child aggression risk in mothers and fathers: Role of emotion regulation and frustration tolerance. Journal of Child and Family Studies, 26(9), 2529-2538. https://doi.org/10.1007/s10826-017-0764-y

Rodriguez, C. M, Russa, M. B., \& Kircher, J. C. (2015). Analog assessment of frustration tolerance: Association with self-reported child abuse risk and physiological reactivity. Child Abuse and Neglect, 46, 121-131. https://doi.org/ 10.1016/j.chiabu.2015.02.017

Sege, R. D., \& Amaya-Jackson, L. (2017). Clinical considerations related to the behavioral manifestations of child maltreatment. Pediatrics, 139(4). https://doi.org/10.1542/peds.2017-0100

Smith, B. L. (2012). The case against spanking [Artigo em newsletter]. American Psychological Association. http://www.apa.org/monitor/2012/04/spanking.aspx

Taillieu, T. L., Afifi, T. O., Mota, N., Keyes, K. M., Sareen, J. (2015). Age, sex, and racial differences in harsh physical punishment: Results from a nationally representative United States sample. Child Abuse and Neglect, 38(12), 1885-1894. https://doi.org/10.1016/j.chiabu.2014.10.020

Venta, A., Velez, L., \& Lau, J. (2016). The role of parental depressive symptoms in predicting dysfunctional discipline among parents at high-risk for child maltreatment. Journal of Child and Family Studies, 25(10), 3076-3082. https://doi.org/10.1007/s10826-016-0473-y

World Health Organization \& International Society for Prevention of Child Abuse and Neglect. (2006). Preventing child maltreatment: A guide to taking action and generating evidence. World Health Organization. 


\section{Roberta Noronha Azevedo}

Psicóloga, Mestra em Ciências pela Faculdade de Filosofia, Ciências e Letras de Ribeirão Preto, Universidade de São Paulo (USP), Ribeirão Preto - SP. Brasil.

E-mail: rn_azevedo@yahoo.com.br

(D) https://orcid.org/0000-0002-3893-8112

Marina Rezende Bazon

Professora Associada do Departamento de Psicologia da Faculdade de Filosofia, Ciências e Letras de Ribeirão Preto, Universidade de São Paulo (USP), Ribeirão Preto - SP. Brasil.

E-mail: mbazon@ffclrp.usp.br

(iD) https://orcid.org/0000-0002-8037-8710

Endereço para correspondência:

Universidade de São Paulo. Faculdade de Filosofia, Ciências e Letras de Ribeirão Preto. Departamento de Psicologia. Av. Bandeirantes, 3900, Monte Alegre. CEP: 14040-901. Ribeirão Preto - SP. Brasil.

Recebido $12 / 07 / 2018$

Aceito 06/04/2020

Received 07/12/2018

Approved 04/06/2020

Recibido 12/07/2018

Aceptado 06/04/2020

Como citar: Azevedo, R. N., \& Bazon, M. R. (2021). Pais/cuidadores com e sem histórico de abuso: Punições corporais e características psicológicas. Psicologia: Ciência e Profissão, 41 (n.spe 3), 1-16. https://doi.org/10.1590/ 1982-3703003207756

How to cite: Azevedo, R. N., \& Bazon, M. R. (2021). Parents/caregivers with and without history of abuse: Corporal punishment and psychological characteristics. Psicologia: Ciência e Profissão, 41 (n.spe 3), 1-16. https://doi.org/ 10.1590/1982-3703003207756

Cómo citar: Azevedo, R. N., \& Bazon, M. R. (2021). Padres/cuidadores con y sin antecedentes de maltrato: Castigos corporales y características psicológicas. Psicologia: Ciência e Profissão, 41 (n.spe 3), 1-16. https://doi.org/ $10.1590 / 1982-3703003207756$ 